\title{
Hyperglycemia and Its Impact on Length of Stay in Patients Hospitalized with Community-Acquired Pneumonia
}

\section{Muhammad Nazeer, ${ }^{1}$ Muhammad Naeem Afzal, ${ }^{2}$ Tabish Raza, ${ }^{3}$ Sidra Rasheed, ${ }^{4}$ Tariq Suleman, Muhammad Arif Nadeem ${ }^{6}$}

\section{Abstract \\ Objectives:}

1. To determine the frequency of hyperglycemia in hospitalized patients with community acquired pneumonia

2. To compare mean hospital stay in patients with and without hyperglycemia admitted with CAP.

Methods: It was a descriptive, case series done at Department of Medicine, Services Hospital, Lahore, in 2016. With non-probability, consecutive sampling technique a sample size of 150 cases is calculated with $95 \%$ confidence level, $8 \%$ margin of error and talking expected percentage of hyperglycemia as $38.2 \%$ in hospitalized patients with community acquired pneumonia.

Results: Out of total 150 cases of CAP there were $85(56.67 \%)$ males with mean age of $47.61 \pm 14.66$ years. Hyperglycemia was seen in $46(30.67 \%)$ patients with CAP. There was equal distribution of hyperglycemia with respect to gender and age groups with $p=0.56$ and 0.24 respectively. Hyperglycemia was more in those having temperature more than $1010 \mathrm{~F}$ however this difference was not significant with $\mathrm{p}=0.32$. There was significant difference seen in terms of length of hospital stay, $4.07 \pm 1.51$ days in hyperglycemic and $2.85 \pm 1.31$ days in normoglycemics with $p=0.001$. The length of hospital stay was near significant high in males, $4.31 \pm 1.56$ days $(p=0.08)$, while non -significant in females with $p=0.30$. There was no significant difference in terms of age groups in cases with or without hyperglycemia with $p=0.56$ and 0.78 . The length of hospital stay was longer in those that had temperature more than $101 \mathrm{oF}, 4.29 \pm 1.26$ days with hyperglycemia as compared to $2.88 \pm 1.34$ days in normoglycemics with $\mathrm{p}=0.13$.

Conclusion: Community acquired pneumonia is an important infectious health concern. It is associated with high blood glucose and the length of hospital stay is found significantly high in cases of hyperglycemia.

Key words: Community acquired pneumonia, Hyperglycemia, hospital stay

\section{Introduction}

Oommunity acquired pneumonia (CAP) is a major cause of morbidity and mortality in low

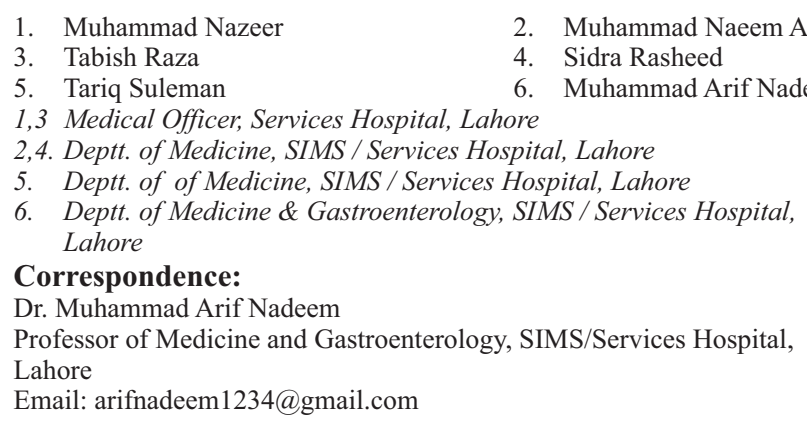

Email: arifnadeem1234@gmail.com

Submission Date: $\quad$ 08-03-2020

1st Revision Date: $\quad 08-09-2020$

Acceptance Date: $\quad 26-10-2020$ and middle income countries. In the last decade, there have been several advances and new interventions resulting in a substantial reduction in pneumonia incidence and its improved outcomes. ${ }^{1}$ Hyperglycemia has been established as an independent predictor of morbidity and mortality in patients admitted with acute coronary syndromes, ischemic stroke, trauma, heart failure, and coronary artery bypass graft, as well as admission to surgical and medical intensive care units ${ }^{2}$. Although hyperglycemia leads to impaired host immune responses, its impact on the outcomes (e.g. mortality, length of stay (LOS), and readmission) is not clear in CAP. The relative impact of glycemic control on LOS among CAP patients has not been well established. Evidence that hyperglycemia affects the outcomes suggests a need for its evaluation in 
the context of $\mathrm{CAP}$ - hospitalized patients. ${ }^{1,2}$

Appropriate initial management of pneumonia and subsequently the outcomes depend on an accurate assessment of disease severity. To support clinical assessment, several severity-scoring indices like CURB 65 (confusion, urea, respiratory rate, blood pressure, and 65 years of age or older) or qSOFA score (quick sequential organ failure assessment) on presentation have been developed, which facilitate the prompt identification of individuals with severe disease and allowing rapid institution of intensive care interventions. ${ }^{2}$ However, many such tools may actually underestimate disease severity in young people $^{1,2}$. Similarly, differences in population comorbidity burden and etiological spectrum of pneumonia may reduce the prognostic performance of most pneumonia severity assessment tool that were almost exclusively developed and validated in well - resourced setting. ${ }^{1,2}$ There is an urgent need for the development of validated severity assessment tools that can support clinical management decisions in low socioeconomic countries like Pakistan. Glycemic status at admission is readily available for all patients, and might provide a potentially useful predictive factor for outcomes and utilization of care. ${ }^{3}$

\section{Objectives of the Study}

1. To find frequency of hyperglycemia in hospitalized patients with community acquired pneumonia

2. To compare mean hospital stay in patients with and without hyperglycemia admitted with CAP.

\section{Methods}

It was a descriptive, case series done at Department of Medicine, Medical Unit III, Services Hospital, Lahore, in 2016. With non-probability, consecutive sampling technique a sample size of 150 cases was calculated with $95 \%$ confidence level, $8 \%$ margin of error and taking expected percentage of hyperglycemia as $38.2 \%$ in hospitalized patients with community acquired pneumonia.

\section{Inclusion Criteria}

- All patients hospitalized with CAP aged between 17 and 80 years of both genders.

\section{Exclusion Criteria}

- Antibiotics initiated for respiratory symptoms within 3 weeks prior to hospitalization.

- Hospital discharge $<30$ days preceding present hospital admission date (to rule out nosocomial pneumonia)

- History of suspected aspiration, pulmonary tuberculosis or pneumocystis carinii pneumonia within 1 year prior to admission, use of immunosuppressive agents or steroids

- Acquired Immune Deficiency Syndrome or human immunodeficiency virus infection

- Tracheostomy

- Patients with interstitial lung disease, chronic obstructive airway disease, chronic kidney disease, congestive cardiac failure andpregnancy

\section{Date Collection Procedure}

A total of 150 patients with CAP fulfilling the inclusion and exclusion criteria aged between 17 and 80 years were selected. Bio data was collected after informed consent. Random blood sugar level (BSR) at admission was measured using a glucometer. Patients were labeled as normoglycemia (BSR $<140 \mathrm{mg} / \mathrm{dl}$ ) and hyperglycemia (BSR > 140mg/dl). Temperature record was maintained. Axillary temperature was recorded 12 hourly. Length of stay was calculated for all patients.

\section{Data Analysis Procedure}

Statistical analysis was done using Statistical Package for Social Sciences (SPSS) version 16. Qualitative data like gender and hyperglycemia were presented as frequencies and percentages. Quantitative data i.e. age, blood glucose level and length (duration) of hospital stay were presented as means and standard deviations. Independent sample $t$ test was used to compare the mean hospital stay in patients with or without hyperglycemia. A $p$ value of $\leq 0.05$ was considered as significant. Data was stratified for age and gender to deal with effect modifiers. Post stratification independent sample $t$ test was applied to see the effect of effect modifiers on length of stay and $p$ value of $\leq 0.05$ was considered as significant.

\section{Results}

In this study out of total 150 cases of CAP, 85 
(56.67\%) were males and $65(43.33 \%)$ females, table1. The mean age was $47.61 \pm 14.66$ years while mean BSR, temperature and length of hospital stay were $110.4 \pm 31.61 \mathrm{mg} / \mathrm{dl}, 100.81 \pm 1.58^{\circ} \mathrm{F}$ and $3.22 \pm$ 1.48 days respectively, tables 2 and 3. Eighty (53.33 $\%$ ) cases were having age 50 years or less while 70 (46.67\%) were more than 50 years, tables 4 and 5 . Out of 150 cases, $100(66.67 \%)$ had temperature of $101 \mathrm{oF}$ or less, table 6 . Hyperglycemia (uncontrolled diabetes) was seen in $46(30.67 \%)$ cases of CAP, table 5. There was equal distribution of hyperglycemia with respect to gender and age groups with $\mathrm{p}=0.56$ and 0.24 respectively, table 1and 5. Hyperglycemia was more in cases that had temperature more than $101^{\circ} \mathrm{F}$ however this difference was not significant with $\mathrm{p}=$ 0.32 , table 6 . There was significant difference seen in terms of length of hospital stay i.e., $4.07 \pm 1.51$ days in hyperglycemic and $2.85 \pm 1.31$ days in normoglycemics with $p=0.001$, table 4 . The length of hospital stay was near significant high in males, $4.31 \pm 1.56$ days ( $p=0.08$ ), while it was non-significant in females with $p=0.30$, table 07 . There was no significant difference in terms of age groups in cases with or without hyperglycemia (tables1 \& 4) with $\mathrm{p}=0.56$ and 0.78 . The length of hospital stay was longer in those that had temperature more than $101^{\circ} \mathrm{F}, 4.29 \pm 1.26$ days with hyperglycemia as compared to $2.88 \pm 1.34$ days in normoglycemics with $\mathrm{p}=0.13$, table 6 .

\section{Discussion}

Table 1: Hyperglycemia with Respect to Gender

\begin{tabular}{|c|c|c|c|}
\hline \multirow{2}{*}{ GENDER } & \multicolumn{2}{|c|}{ HYPERGLYCEMIA } & \multirow{2}{*}{ Total } \\
\hline & Yes & No & \\
\hline Male & $26(30.59 \%)$ & $59(69.41 \%)$ & $85(100 \%)$ \\
\hline Female & $20(30.77 \%)$ & $45(69.23 \%)$ & $65(100 \%)$ \\
\hline Total & $46(30.67 \%)$ & $104(69.33 \%)$ & $150(100 \%)$ \\
\hline
\end{tabular}

Chi square $=0.001, p=0.56$

Table 2: Study Variables (Age \& BSR)

\begin{tabular}{lccc}
\hline & & \multicolumn{2}{c}{ VARIABLES } \\
\cline { 3 - 4 } & & Age & BSR \\
\multicolumn{1}{c}{ Mean } & 47.61 & 110.4 \\
\hline 95\%Confidence & Lower Bound & 45.25 & 104.94 \\
Interval for Mean & Upper Bound & 49.98 & 115.14 \\
Median & & 45.00 & 98.00 \\
Std. Deviation & & 14.66 & 31.61 \\
Minimum & 18 & 69 \\
Maximum & & 80 & 190 \\
\hline
\end{tabular}

Community-acquired pneumonia (CAP) is the infection of the lung parenchyma, which is acquired from the organism present in the community. It is a common infection and can be managed in outpatients, in patient and ICUs depending upon the severity of the illness. It is associated with considerable morbidity and mortality, particularly in elderly

Table 3: Study Variables (Duration of Hospital Stay \& Temperature)

\begin{tabular}{lccc}
\hline & \multicolumn{2}{c}{ VARIABLES } \\
\cline { 2 - 4 } & & $\begin{array}{c}\text { Duration of } \\
\text { hospital stay (days) }\end{array}$ & $\begin{array}{c}\text { Temperature } \\
\text { (F) }\end{array}$ \\
\hline \multicolumn{1}{c}{ Mean } & 3.22 & 100.81 \\
95\% Confidence & 2.98 & 45.25 & 100.56 \\
Interval for Mean & 3.46 & 49.98 & 101.07 \\
Median & & 2.00 & 101.00 \\
Std. Deviation & 1.48 & 1.58 \\
Minimum & 2 & 99 \\
Maximum & 8 & 104 \\
\hline
\end{tabular}

Table 4: Stratification for Length of Hospital Stay in Patientswith or without Hyperglycemia with Respect to Age Groups

\begin{tabular}{lcccc}
\hline Age groups & Hyper- & \multicolumn{2}{c}{ Length of hospital stay } & \\
\cline { 3 - 4 } (years) & glycemia & $\mathbf{n}$ & Mean \pm SD & \\
\hline 50 or less & Yes & 27 & $3.81 \pm 1.41$ & 0.09 \\
& No & 53 & $2.32 \pm 0.87$ & \\
$>50$ & Yes & 19 & $4.42 \pm 1.61$ & 0.78 \\
& No & 51 & $3.39 \pm 1.47$ & \\
\hline
\end{tabular}

Normoglycemics with $\mathrm{p}=0.001$

Table 5: Hyperglycemia with Respect to Age Groups

\begin{tabular}{cccc}
\hline \multirow{2}{*}{$\begin{array}{c}\text { AGE GROUPS } \\
\text { (years) }\end{array}$} & \multicolumn{2}{c}{ HYPERGLYCEMIA } & \multirow{2}{*}{ Total } \\
\cline { 2 - 3 } & Yes & No & \\
\hline 50 or less & $27(23.75 \%)$ & $53(66.25 \%)$ & $80(100 \%)$ \\
$>50$ & $19(27.14 \%)$ & $51(72.86 \%)$ & $70(100 \%)$ \\
Total & $\mathbf{4 6 ( 3 0 . 6 7 \% )}$ & $\mathbf{1 0 4}(\mathbf{6 9 . 3 3 \% )}$ & $\mathbf{1 5 0 ( \mathbf { 1 0 0 \% } )}$ \\
\hline
\end{tabular}

Chi square $=0.76, p=0.24$

Table 6: Stratification for Length of Hospital Stay in Patients with or without Hyperglycemia with Respect to Temperature

\begin{tabular}{|c|c|c|c|c|}
\hline \multirow{2}{*}{$\begin{array}{l}\text { Tempe- } \\
\text { rature }\end{array}$} & \multirow{2}{*}{$\begin{array}{c}\text { Hyper- } \\
\text { glycemia }\end{array}$} & \multicolumn{2}{|c|}{ Length of hospital stay } & \multirow{2}{*}{ p value } \\
\hline & & $\mathbf{N}$ & Mean \pm SD & \\
\hline \multirow[t]{2}{*}{101 or less } & Yes & 29 & $3.93 \pm 1.64$ & \multirow[t]{2}{*}{0.41} \\
\hline & No & 71 & $2.83 \pm 1.30$ & \\
\hline \multirow[t]{2}{*}{$>101$} & Yes & 17 & $4.29 \pm 1.26$ & \multirow[t]{2}{*}{0.13} \\
\hline & No & 33 & $2.88 \pm 1.34$ & \\
\hline
\end{tabular}


Table 7: Stratification for Length of Hospital Stay in Patients with or without Hyperglycemia with Respect to Gender

\begin{tabular}{lcccc}
\hline \multirow{2}{*}{ Gender } & $\begin{array}{c}\text { Hyper- } \\
\text { glycemia }\end{array}$ & \multicolumn{2}{c}{ Length of hospital stay } & \\
\cline { 3 - 4 } Male & Yes & 26 & Mean \pm SD & \\
\hline \multirow{4}{*}{ Female } & No & 59 & $2.31 \pm 1.56$ & 0.08 \\
& Yes & 20 & $3.75 \pm 1.41$ & 0.30 \\
& No & 45 & $2.78 \pm 1.27$ & \\
\hline
\end{tabular}

patients and those with significant comorbidities. Various other factors affect the length of hospital stay and pose a huge burden over the patients as well as the health care system of the community. Hyperglycemia was seen in 46 (30.67\%) cases admitted with community acquired pneumonia. Similar pattern was seen in other studies that also had higher number ranging from 30 to $40 \%$. $^{4-7}$

Why the cases in our study had relatively lower number of hyperglycemia as compared to other studies? It may be because this study was conducted in medical wards as compared to other studies which were conducted in pulmonology departments where serious cases are admitted and have a higher chance of getting hyperglycemia. This also reflects the disease process that greater the stress of the disease and higher are the chances of counter regulatory hormones to be released and leading to increased level of glucose.

Hyperglycemia was slightly higher in females where it was seen in in $20(30.77 \%)$ cases as compared to males having $26(30.59 \%)$ cases in their respective groups with $\mathrm{p}$ value of 0.56 . However this difference was not statistically significant. Similar pattern was observed by Sarkar $\mathrm{M}$ and Trifirò $\mathrm{G}$ et al. ${ }^{8-9}$ The higher level of glucose in females can be due to hormonal differences as compared to males and secondly the impact of infections in females lead to more anxiety and again increasing the counter regulatory hormones.

Hyperglycemia in age group of 50 year or more was seen in $19(27.14 \%)$ of cases as compared to 27 $(23.75 \%)$ with age less than this with an insignificant $\mathrm{p}$ value of 0.24 . This was in contrast to few studies done in the past by Laheij RJ et al and Almirall J et al that found this more in the younger age groups. ${ }^{10-11}$ The reason for this higher number in older age group can be explained by the fact that in such cases they get more severe infection and that can be a cause of hyperglycemia as was seen in present study as a marker of severe infection. On the other hand contradicting results can be the involvement of the other mechanisms as well. As in old age the reflex mechanisms are slowed down and on the other hand the chances of serious infection due to immune compromised state of old age can also lead to adrenal crises which can also end up with relatively lower levels of serum sugars.

In cases with temperature more than $101 \mathrm{~F}$, the hyperglycemia was seen in 17 (34\%) of cases in contrast to $29(29 \%)$ with temperature less than this with $p=0.32$. Similar was seen by Wunderink RG et al and Johansson $\mathrm{N}$ et al. ${ }^{12-13}$ This again strengthens the belief that higher the temperature and higher is the stressful condition and more are the chances for glucose to be raised.

Mean hospital stay in males with hyperglycemia was $4.31 \pm 1.56$ days as compared to normoglycemics having $2.90 \pm 1.34$ days with $\mathrm{p}=0.08$ while the difference of this glycemic levels infemales was not significant with $p=0.30$. Similar was seen by Marrie TJ et al Aujesky D et al. ${ }^{14-15}$ They also did not find any significant association with this. The reason of male patients to have prolonged hospital stay can be explained by the presence of co morbid factors like smoking which is not only a risk factor to predispose to infection but also lead to delayed recovery.

A non-significant statistical difference was seen regarding mean length of hospital stay in patients with or without hyperglycemia in both the age groups; however this difference was more seen in cases that had age 50 years or less having stay of $3.81 \pm 1.41$ days as compared to normoglycemics having stay of $2.32 \pm$ 0.87 days with $p$ value of 0.56 . Moreover, the overall stay was higher in cases with age groups more than 50 years. Similar trends were seen in a study done by Suter-Widmer I et $\mathrm{al}^{16}$ who also had longer hospital stay in their study with significant $p$ value of 0.001 but the cute off value for age in their study was 60 years. However, studies conducted by Kaysar $\mathrm{M}$ et al and Labarere $\mathrm{J}$ et al did not reveal any significant association but still the cases with higher age groups had a longer hospital stay just like our study. ${ }^{17-18}$ Why the cases with higher age had a longer stay because of the 
multiple factors like at older age the immunity is suppressed due to reasons like malignancies, malabsorption or other co morbid conditions which add up to the primary diagnosis of CAP and lead to a further stay. Moreover the patients with old age can also be socially deprived and factors like good care at hospital can also prolong their stay. In contrast to this the younger ones with good immunity and mental strength to cope up with infection lead to shorter hospital stay.

There was significant difference seen in terms of length of hospital stay where it was $4.07 \pm 1.51$ days in hyperglycemic and $2.85 \pm 1.31$ days in normoglycemics with $p=0.001$. Similar was seen by a study done by Godar DA et al who found mean hospital stay of 4.2 days as compared to 3.9 with normal glucose level which was found statistically significant with $p$ value of $0.04 .^{19}$ Studies done by McAlister FA et al and Latham $\mathrm{R}$ et al who also had it more in patients with hyperglycemia though this difference was not statistically signifcatn. ${ }^{20-21}$ Why the length of stay was longer with hyperglycemia? It can be due to severity of the disease, which was not measured by any score in this study. The association of multiple factors like high glucose, high temperature and longer hospital stay all reflect a severer form of disease process. Moreover the effects of hyperglycemia like weakness and polyuria also lead to extra set of symptoms to the previously suffering patients with symptoms of pneumonia and lead to further delay in discharge.

There were many strengths of this study. Considering the burden posed by CAP patients in medical wards, no study was carried out. Moreover we evaluated the factor of BSR which is recently worked up to look for its impact and this study also assessed the wide range of age group.

However there were few limitations of this study as well. The cases were not assessed for the severity of pneumonia over any score hence it was difficult to assess which group had the more severe cases. Furthermore the co-morbid conditions like diabetes mellitus, hypertensionand malignancies were not assessed.

Further studies with larger number of patients, better description of pneumonia severity and with comorbid conditions can explore the impact of hyper- glycemia in length of hospital stay.

\section{Conclusion}

Community acquired pneumonia is an important infectious health concern. It is associated with high blood glucose and the length of hospital stay is found significantly high in cases of hyperglycemia.

\section{Author's Contribution}

MN: Data Collection, Statistical Analysis, Manuscript writing

MNA: Write up, Statistical Analysis

TR: SR: Statistical Analysis

TS: Editing, Review

MAN: Designed, Writing, Editing

\section{References}

1. Zar HJ, Madhi SA, Aston SJ, Gordon SB. Pneumonia in low and middle income countries: progress and challenge. Thorax. 2013;68(11):1052-6.

2. Murad MH et al. Glycemic control in on critically ill hospitalized patients: a systematic review and metaanalysis. J Clin Endocrinol Metab. 2012; 97(1):4958.

3. Bhattacharya RK, Mahnken JD, Rigler SK. Impact of admission blood glucose level on outcomes in community acquired pneumonia in older adults. Int $\mathrm{J}$ Gen Med. 2013; 6:341-4.

4. Bhattacharya RK, Mahnken JD, Rigler SK. Impact of admission blood glucose level on outcomes in community acquired pneumonia in older adults. Int $\mathrm{J}$ Gen Med. 2013;6:341

5. Godar DA et al. the impact of serum glucose on clinical outcome in patients hospitalized with community-acquired pneumonia. WMJ. 2011; 110(1):14-20.

6. Castellanos MR et al. Fasting hyperglycemia upon hospital admission is associated with higher pneumonia complication rated among the elderly. Int Arch Med. 2010; 3:16.

7. Lepper PM et al, German Community Acquired Pneumonia Competence Network. Serum glucose levels for predicting death in patients admitted to hospital for community acquired pneumonia: prospective cohort study. BM. 2010; 344:3397.

8. Sarkar M, Hennessy S, Yang YX. Proton-pump inhibitor use and the risk for community-acquired pneumonia. Ann Intern Med 2008; 149:391.

9. Trifirò G, Gambassi G, Sen EF, et al. Association of community-acquired pneumonia with antipsychotic drug use in elderly patients: a nested case-control study. Ann Intern Med 2010; 152:418. 
10. Almirall J, Bolíbar I, Balanzó X, González CA. Risk factors for community-acquired pneumonia in adults: a population-based case-control study. Eur Respir J 1999; 13:349.

11. Laheij RJ, Sturkenboom MC, Hassing RJ, et al. Risk of community-acquired pneumonia and use of gastric acid-suppressive drugs. JAMA 2004; 292:1955.

12. Wunderink RG, Waterer GW. Community-acquired pneumonia: pathophysiology and host factors with focus on possible new approaches to management of lower respiratory tract infections. Infect Dis Clin North Am 2004; 18:743.

13. Johnson N, Kalin M, Tiveljung-Lindell A, et a. Etiology of community-acquired pneumonia: increased microbiological yield with new diagnostic methods. Clin Infect Dis 2010; 50:202-209.

14. Marrie $\mathrm{TJ}, \mathrm{Wu}$ L. Factors influencing in-hospital mortality in community-acquired pneumonia: a prospective study of patients not initially admitted to the ICU. Chest 2005; 127:1260.

15. Aujesky D, Auble TE, Yealy DM, et al. Prospective comparison of three validated prediction rules for prognosis in community-acquired pneumonia. Am J Med 2005; 118:384.

16. Suter-Widmer I, Christ-Crain M, Zimmerli W, Albrich W, Mueller B, Schuetz P. Predictors for length of hospital stay in patients with communityacquired Pneumonia: Results from a Swiss Multicenter study. BMC Pulmonary Medicine201212:21.

17. Kaysar M, Augustine T, Jim L, Benjamin C: Predictors of length of stay between the young and aged in hospitalized community-acquired pneumonia patients. Geriatric Gerontol Int. 2008, 8 (4): 227-233.

18. Labarere J, Stone RA, Scott OD, et al: Factors associated with the hospitalization of low-risk patients with community-acquired pneumonia in a clusterrandomized trial. J Gen Intern Med. 2006, 21 (7): 745-752.

19. Godar DA, Kumar DR, Schmelzer KM, Ta SR. The Impact of Serum Glucose on Clinical Outcomes in Patients Hospitalized with Community-Acquired Pneumonia. Wiscon Med J. 2011; 110(1):8-20.

20. McAlister FA, Majumdar SR, Blitz S, Rowe BH, Romney J, Marrie TJ. The relation between hyperglycemia and outcomes in 2471 patients admitted to the hospital with community-acquired pneumonia. Diabetes Care. 2005; 28(4):810-815.

21. Latham R, Lancaster AD, Covington JF, Pirolo JS, Thomas CS. The association of diabetes and glucose control with surgical-site infection among cardiothoracic surgery patients. Infect Control Hosp Epidemiol. 2001;22(10):607-612. 